\title{
Noncontact friction between nanostructures
}

\author{
A. I. Volokitin ${ }^{1,2}$ and B. N. J. Persson ${ }^{1}$ \\ ${ }^{1}$ Institut für Festkörperforschung, Forschungszentrum Jülich, D-52425, Germany \\ ${ }^{2}$ Samara State Technical University, 443100 Samara, Russia
}

(Received 16 January 2003; revised manuscript received 30 May 2003; published 21 October 2003)

\begin{abstract}
We calculate the van der Waals friction between two semi-infinite solids in normal relative motion and find a drastic difference in comparison with the parallel relative motion. The case of good conductors is investigated in detail both within the local optic approximation and using a nonlocal optic dielectric approach. We show that the friction may increase by many orders of magnitude when the surfaces are covered by adsorbates, or can support low-frequency surface plasmons. In this case the friction is determined by resonant photon tunneling between adsorbate vibrational modes, or surface plasmon modes. The theory is compared to atomic force microscope experimental data.
\end{abstract}

DOI: 10.1103/PhysRevB.68.155420

PACS number(s): 73.61.- $-\mathrm{r}$

\section{INTRODUCTION}

A great deal of attention has been devoted to noncontact friction between nanostructures, including, for example, the frictional drag force between two-dimensional quantum wells, ${ }^{1-4}$ and the friction force between an atomic force microscope tip and a substrate. ${ }^{5-9}$

In noncontact friction the bodies are separated by a potential barrier thick enough to prevent electrons or other particles with a finite rest mass from tunneling across it, but allowing interaction via the long-range electromagnetic field, which is always present in the gap between bodies. The presence of inhomogeneous tip-sample electric field is difficult to avoid, even under the best experimental conditions. ${ }^{7}$ For example, even if both the tip and sample were metallic single crystals, the tip would still have corners present and more than one crystallographic plane exposed. The presence of atomic steps, adsorbates, and other defects will also contribute to the inhomogeneous electric field. The electric field can be easily changed by applying a voltage between the tip and the sample.

The electromagnetic field can also be created by the fluctuating current density, due to thermal and quantum fluctuations inside the solids. This fluctuating electromagnetic field is always present close to the surface of any body, and consists partly of traveling waves and partly of evanescent waves which decay exponentially with the distance away from the surface of the body. The fluctuating electromagnetic field originating from the fluctuating current density inside the bodies gives rise to the well-known long-range attractive van der Waals interaction between two bodies. ${ }^{10}$ If the bodies are in relative motion, the same fluctuating electromagnetic field will give rise to a friction which is frequently named the van der Waals friction. Van der Waals friction can be considered to be mediated by photon exchange between the bodies: One body emit a photon, and the other absorbs it, thus transferring momentum between the bodies, resulting in a friction force. At large distances between the bodies, the main contribution to the friction comes from photon exchange, corresponding to the propagating electromagnetic waves. However, this contribution is very small because the photons corresponding to propagating waves carry a very small mo- mentum, no larger than $k_{B} T / c$. The photons, corresponding to the evanescent electromagnetic waves, carry the momentum $<\hbar d^{-1}$. Thus for distances $d$ between two bodies smaller than the characteristic distance $d_{T}=\hbar c / k_{B} T$, which depends on temperature (at room temperature $d_{T} \sim 10^{5} \AA$ ), the main contribution to the friction comes from the evanescent electromagnetic field. In analogy with electron tunneling, this mechanism of momentum transfer can be considered to be associated with the photon tunneling.

Although the dissipation of energy connected with the noncontact friction is always of electromagnetic origin, the detailed mechanism is not totally clear, since there are several different mechanisms of energy dissipation connected with the electromagnetic interaction between bodies. First, the electromagnetic field from one body will penetrate into the other body, and induce an electric current. In this case friction is due to ohmic losses inside the bodies. The fluctuating electromagnetic field can also excite the vibrations of the adsorbates or other surface localized modes, e.g., surface plasmons and polaritons. In this case friction is due to energy relaxation of the surface modes. Another contribution to friction from the electromagnetic field is associated with the time-dependent stress acting on the surface of the bodies. This stress can excite acoustic waves, or induce timedependent deformations which may result in a temperature gradient. It can also induce motion of defects either in the bulk, or on the surface of the bodies. The contribution to friction due to nonadiabatic heat flow, or motion of defects, is usually denoted as internal friction.

It is very worthwhile to get a better understanding of different mechanisms of noncontact friction because of it practical importance for ultrasensitive force detection experiments. This is because the ability to detect small forces is inextricably linked to friction via the fluctuation-dissipation theorem. For example, the detection of single spins by magnetic resonance force microscopy, which has been proposed for three-dimensional atomic imaging ${ }^{11}$ and quantum computation, ${ }^{12}$ will require force fluctuations to be reduced to unprecedented levels. In addition, the search for quantum gravitation effects at short length scale ${ }^{13}$ and future measurements of the dynamical Casimir forces ${ }^{14}$ may eventually be limited by noncontact friction effects. 
Recently Gotsmann and Fuchs ${ }^{6}$ observed long-range noncontact friction between an aluminum tip and a gold (111) surface. The friction force $F$ acting on the tip is proportional to the velocity $v, F=\Gamma v$. For motion of the tip normal to the surface the friction coefficient $\Gamma(d)=b \cdot d^{-3}$, where $d$ is the tip-sample spacing and $b=\left(8.0_{-4.5}^{+5.5}\right) \times 10^{-35} \mathrm{~N} \mathrm{~s} \mathrm{~m}^{2}{ }^{6}$ Later Stipe et al. ${ }^{7}$ observed a noncontact friction effect between a gold surface and a gold-coated cantilever as a function of the tip-sample spacing $d$, the temperature $T$, and the bias voltage $V$. For vibration of the tip parallel to the surface they found $\Gamma(d)=\alpha(T)\left(V^{2}+V_{0}^{2}\right) / d^{n}$, where $n=1.3 \pm 0.2$ and $V_{0}$ $\sim 0.2 \mathrm{~V}$. At $295 \mathrm{~K}$, for the spacing $d=100 \AA$ they found $\Gamma$ $=1.5 \times 10^{-13} \mathrm{kgs}^{-1}$, which is $\sim 500$ times smaller that reported in Ref. 6 at the same distance using a parallel cantilever configuration.

In a recent paper, Dorofeev et al. ${ }^{5}$ claimed that the noncontact friction effect observed in Refs. 5 and 6 is due to Ohmic losses mediated by the fluctuating electromagnetic field. This result is controversial, however, since the van der Waals friction has been shown ${ }^{15-18}$ to be many orders of magnitude smaller than the friction observed by Dorofeev et al. Presently, the origin of the difference in magnitude and distance dependence of the long-range noncontact friction effect observed in Refs. 6 and 7 is not well understood.

In order to improve the basic understanding of noncontact friction, we present results for the van der Waals friction. In Ref. 15 we developed a theory of van der Waals friction for surfaces in parallel relative motion. Here we generalize the theory also to include the case when the surfaces are in normal relative motion, and we show that there is a drastic difference between these two cases. Thus, for normal relative motion of clean good conductor surfaces, the friction is many orders of magnitude larger than for parallel relative motion, but still smaller than observed experimentally. Another enhancement mechanism of the noncontact friction can be connected with resonant photon tunneling between states localized on the different surfaces. Recently it was discovered that resonant photon tunneling between surface plasmon modes give rise to an extraordinary enhancement of the optical transmission through subwavelength hole arrays. ${ }^{19}$ The same surface modes enhancement can be expected for van der Waals friction if the frequency of these modes is sufficiently low to be excited by thermal radiation. At room temperature only modes with frequencies below $\sim 10^{13} \mathrm{~s}^{-1}$ can be excited. For normal metals surface plasmons have much too high frequencies; at thermal frequencies the dielectric function of normal metals becomes nearly purely imaginary, which exclude a surface plasmon enhancement of the van der Waals friction for good conductors. However, surface plasmons for semiconductors are characterized by much smaller frequencies and damping constants, and they can give an important contribution to van der Waals friction. Other surface modes which can be excited by thermal radiation are adsorbate vibrational modes. Especially for parallel vibrations these modes may have very low frequencies.

All information about the long-range electromagnetic interaction between two noncontacting bodies is, in principle, contained in the reflection factors of the electromagnetic field. At the present time very little is known about the re- flection factors for large wave vectors and for extremely small frequencies. In our previous calculations of the van der Waals friction ${ }^{15-18}$ we mostly considered good conductors. In this case it was shown that the important contribution comes from the nonlocal optic effects in the surface region. However, it was shown that the van der Waals friction becomes much larger for a high resistivity material, for which the volume contribution from nonlocal effects is also important. Nonlocal optics refer to the fact that the current at point $\mathbf{r}$ depends on the electric field not only at point $\mathbf{r}$, as it is assumed within local optic approximation, but also at points $\mathbf{r}^{\prime} \neq \mathbf{r}$ in a finite region around the point $\mathbf{r}$. In the case when both points are located outside the surface region the dielectric response function can be expressed through the dielectric function appropriate for a semi-infinite electron gas. However, if one of the point $\mathbf{r}$ or $\mathbf{r}^{\prime}$ is located in the surface region, the dielectric response function will be different from its volume value, and this gives a surface contribution from nonlocality. It is easy to see that within the local optic approximation the van der Waals friction diverge when the conductivity of materials tend to zero. This means that the local optic approximation breaks down for high-resistivity materials. This situation is completely different from the heat transfer between bodies via photon tunneling, ${ }^{17}$ where the heat flux is maximal at conductivities corresponding to semimetals. In order to clarify the situation we study the dependence of the van der Waals friction on the dielectric properties of the materials within the nonlocal dielectric approach, which was proposed some years ago for an investigation of the anomalous skin effects. ${ }^{20}$

\section{CAlCUlation OF THE FluCtuating ELECTROMAGNETIC FIELD}

We consider two semi-infinite metals 1 and $\mathbf{2}$ having parallel flat surfaces. We introduce a coordinate system with the $x y$ plane in the surface of body $\mathbf{1}$, and the $z$ axis along the upward normal. The surface of body $\mathbf{2}$ is located at $z=d$, performing small amplitude vibrations along the $z$ axes with displacement coordinate $u_{z}(t)=u_{0} e^{-i \omega_{0} t}$. Since the system is translation invariant in the $\mathbf{x}=(x, y)$ plane, the electromagnetic field can be represented by the Fourier integrals

$$
\begin{aligned}
& \mathbf{E}(\mathbf{x}, z)=\int \frac{d^{2} q}{(2 \pi)^{2}} e^{i \mathbf{q} \cdot \mathbf{x}} \mathbf{E}(\mathbf{q}, z), \\
& \mathbf{B}(\mathbf{x}, z)=\int \frac{d^{2} q}{(2 \pi)^{2}} e^{i \mathbf{q} \cdot \mathbf{x}} \mathbf{B}(\mathbf{q}, z),
\end{aligned}
$$

where $\mathbf{E}$ and $\mathbf{B}$ are the electric and magnetic induction fields, and $\mathbf{q}$ is the two-dimensional wave vector in the $(x, y)$ plane. After Fourier transformation it is convenient to choose the coordinate axis in the $(x, y)$ plane along the vectors $\mathbf{q}$ and $\mathbf{n}$ $=[\hat{z} \times \mathbf{q}]$. The scattering of the electromagnetic wave with the frequency $\omega$ on the vibrating surface of body 2 will give rise to the harmonics at the frequencies $\omega \pm \omega_{0}$. Thus in the vacuum gap between the bodies the electric field $\mathbf{E}(\mathbf{q}, \omega, z)$ can, to linear order in the displacement coordinate, be written in the form 


$$
\begin{aligned}
\mathbf{E}(\mathbf{q}, \omega, z)= & {\left[\left(\mathbf{w}_{0} e^{i p z}+\mathbf{v}_{0} e^{-i p z}\right)+\left(\mathbf{w}_{1} e^{i p^{+} z}\right.\right.} \\
& \left.\left.+\mathbf{v}_{1} e^{-i p^{+} z}\right) e^{-i \omega_{0} t}\right] e^{-i \omega t} .
\end{aligned}
$$

From Eq. (3) and the Maxwell equation

$$
-\frac{1}{c} \frac{\partial \mathbf{B}}{\partial t}=\boldsymbol{\nabla} \times \mathbf{E},
$$

we get the magnetic induction field $\mathbf{B}(\mathbf{q}, \omega, z)$,

$$
\begin{aligned}
\mathbf{B}(\mathbf{q}, \omega, z)= & c\left[\frac{1}{\omega}\left(\left[\mathbf{k}^{-1} \times \mathbf{v}_{0}\right] e^{-i p z}+\left[\mathbf{k}^{+} \times \mathbf{w}_{0}\right] e^{i p z}\right)\right. \\
& +\frac{1}{\omega+\omega_{0}}\left(\left[\mathbf{k}_{+}^{-} \times \mathbf{v}_{1}\right] e^{-i p^{+} z}\right. \\
& \left.\left.+\left[\mathbf{k}_{+}^{+} \times \mathbf{w}_{1}\right] e^{i p^{+} z}\right) e^{-i \omega_{0} t}\right] e^{-i \omega t},
\end{aligned}
$$

where $\mathbf{k}^{ \pm}=\mathbf{q} \pm \hat{z} p, \quad p=\left((\omega / c)^{2}-q^{2}\right)^{1 / 2}, \quad p^{+}=p\left(\omega+\omega_{0}\right)$, $\mathbf{k}_{+}^{+}=\mathbf{k}^{+}\left(\omega+\omega_{0}\right)$, and $\mathbf{k}_{+}^{-}=\mathbf{k}^{-}\left(\omega+\omega_{0}\right)$. In Eqs. (3) and (5) $w_{0}, v_{0}$, etc. are unknown amplitudes which are to be found using appropriate boundary conditions (see below). At the surfaces of the bodies the amplitude of the outgoing electromagnetic wave must be equal to the amplitude of the reflected wave plus the amplitude of the radiated wave. It is convenient to decompose the electromagnetic field into $p$ and $s$-polarized electromagnetic waves. For the $p$-polarized electromagnetic waves the electric field is in the incident plane determined by the vectors $\mathbf{q}$ and $\mathbf{n}$, and for the $s$-polarized electromagnetic waves the electric field is normal to the incident plane. Thus the boundary conditions for the electromagnetic field at $z=0$ can be written in the forms

$$
\begin{gathered}
w_{0 z(y)}=R_{1 p(s)}(\omega) v_{0 z(y)}+E_{1 z(y)}^{f}(\omega), \\
w_{1 z(y)}=R_{1 p(s)}\left(\omega+\omega_{0}\right) v_{1 z(y)},
\end{gathered}
$$

where $R_{1 p(s)}(\omega)$ is the reflection amplitude for surface $\mathbf{1}$ for the $p(s)$-polarized electromagnetic field, and where $E_{1 z(y)}^{f}(\omega)$ are the components of the fluctuating electric field outside surface $\mathbf{1}$ in the absence of body $\mathbf{2}$. The boundary condition at the surface of body $\mathbf{2}$ must be written in the reference frame where body $\mathbf{2}$ is at rest. The electric field in this reference frame is determined by a Lorentz transformation. Performing a Lorentz transformation to linear order in $\omega_{0}$ gives

$$
\mathbf{E}^{\prime}=\mathbf{E}-\frac{i \omega_{0} u(t)\left[\hat{\mathbf{e}}_{z} \times \mathbf{B}\right]}{c} .
$$

For the $p$-polarized electromagnetic waves the second term in Eq. (8) is of the order of magnitude $\omega_{0} u_{0} \omega / p c^{2}$ relative to the first one and can be neglected for the most practical cases. However, for the $s$-polarized electromagnetic waves the second term is of the order of magnitude $\omega_{0} u_{0} p / \omega$, and can be of the same order of magnitude as the first term. In the rest frame of body $\mathbf{2}$ there is also a mixture of $s$ - and $p$-polarized electromagnetic waves. In Ref. 15 it was shown that this gives a contribution of the order $\left(\omega_{0} u_{0} / c\right)^{2}$, and thus can be neglected. After performing Lorentz transformation to linear order in $\omega_{0}$ and $u_{0}$ we get $\mathbf{v}_{0}^{\prime}=\mathbf{v}_{0}$ and $\mathbf{w}_{0}^{\prime}$ $=\mathbf{w}_{0}$ :

$$
\begin{gathered}
v_{1 z(x)}^{\prime}=v_{1 z(x)}-i p u_{0} v_{0 z(x)}, \quad w_{1 z(x)}^{\prime}=w_{1 z(x)}+i p u_{0} w_{0 z(x)}, \\
w_{1 y}^{\prime}=w_{1 y}+\frac{\omega+\omega_{0}}{\omega} i p u_{0} w_{0 y}, \quad v_{1 y}^{\prime}=v_{1 y}-\frac{\omega+\omega_{0}}{\omega} i p u_{0} v_{0 y} .
\end{gathered}
$$

The boundary conditions for the electromagnetic field at $z$ $=d+u(t)$ in the rest frame of body $\mathbf{2}$ can be written in the form

$$
\begin{gathered}
v_{0 z(y)}=e^{2 i p d} R_{2 p(s)}(\omega) w_{0 z(y)}+e^{i p d} E_{2 z(y)}^{f}(\omega) \\
v_{1 z}-i p u_{0} v_{0 z}=e^{2 i p^{+} d} R_{2 p}\left(\omega+\omega_{0}\right)\left(w_{1 z}+i p u_{0} w_{0 z}\right) \\
v_{1 y}-i p u_{0} \frac{\left(\omega+\omega_{0}\right) v_{0 y}}{\omega} \\
=e^{2 i p d} R_{2 s}\left(\omega+\omega_{0}\right)\left(\omega_{1 y}+i p u_{0} \frac{\left(\omega+\omega_{0}\right) w_{0 y}}{\omega}\right)
\end{gathered}
$$

where $R_{2 p(s)}(\omega)$ is the reflection amplitude for surface 2 for a $p^{-}(s-)$ polarized electromagnetic field, and where $E_{2 z(y)}^{f}(\omega)$ are the components of the fluctuating electric field outside surface 2 in the absence of body 1. From Eqs. (6) and (7) and (9)-(11) we get

$$
\begin{gathered}
w_{0 z(y)}=\frac{R_{1 p(s)} E_{2 z(y)}^{f} e^{i p d}+E_{1 z(y)}^{f}}{\Delta}, \\
v_{0 z(y)}=\frac{e^{2 i p d} R_{2 p(s)} E_{1 z(y)}^{f}+E_{2 z(y)}^{f} e^{i p d}}{\Delta},
\end{gathered}
$$


where $R_{p(s)}^{+}=R_{p(s)}\left(\omega+\omega_{0}\right), \quad \Delta_{p(s)}=1-e^{2 i p d} R_{2 p(s)} R_{1 p(s)}$, and $\Delta_{p(s)}^{+}=\Delta_{p(s)}\left(\omega+\omega_{0}\right)$. The other components of the fluctuating electromagnetic field can be found from the transversality conditions

$$
q w_{x}+p w_{z}=0, \quad q v_{x}-p v_{z}=0 .
$$

The fundamental characteristic of the fluctuating electromagnetic field is the correlation function, determining the average product of components $\mathbf{E}^{f}(\mathbf{q}, \omega)$. According to the general theory of the fluctuating electromagnetic field (see, for example, Ref. 17) these correlation functions are given by

$$
\begin{aligned}
\left\langle\left|E_{y}^{f}(\mathbf{q}, \omega)\right|^{2}\right\rangle= & \frac{\hbar \omega^{2}}{2 c^{2}|p|^{2}}\left(n(\omega)+\frac{1}{2}\right)\left[\left(p+p^{*}\right)\left(1-\left|R_{s}\right|^{2}\right)\right. \\
& \left.+\left(p-p^{*}\right)\left(R_{s}^{*}-R_{s}\right)\right], \\
\left\langle\left|E_{z}^{f}(\mathbf{q}, \omega)\right|^{2}\right\rangle= & \frac{\hbar q^{2}}{2|p|^{2}}\left(n(\omega)+\frac{1}{2}\right)\left[\left(p+p^{*}\right)\left(1-\left|R_{p}\right|^{2}\right)\right. \\
& \left.+\left(p-p^{*}\right)\left(R_{p}^{*}-R_{p}\right)\right],
\end{aligned}
$$

where $\langle\cdots\rangle$ denotes a statistical average over the random field, and where the Bose-Einstein factor is

$$
n(\omega)=\frac{1}{e^{\hbar \omega / k_{B} T}-1} .
$$

We note that $p$ is real for $q<\omega / c$ (propagating waves), and purely imaginary for $q>\omega / c$ (evanescent waves). Thus for $q<\omega / c$ and $q>\omega / c$ the correlation functions are determined by the first and second terms in Eqs. (18) and (19), respectively.

\section{CALCULATION OF THE FRICTION FORCE BETWEEN TWO SEMI-INFINITE BODIES IN NORMAL RELATIVE MOTION}

The frictional stress $\sigma$ which act on the surfaces of the two bodies can be obtained from the $z z$-component of the Maxwell stress tensor $\sigma_{i j}$, evaluated at $z=0$ :

$$
\begin{aligned}
\sigma_{z z}= & \frac{1}{4 \pi} \int_{0}^{\infty} d \omega \int \frac{d^{2} q}{(2 \pi)^{2}}\left[\left\langle\left|E_{z}(\mathbf{q}, \omega, z)\right|^{2}\right\rangle+\left\langle\left|B_{z}(\mathbf{q}, \omega, z)\right|^{2}\right\rangle\right. \\
& -\left\langle\left|E_{x}(\mathbf{q}, \omega, z)\right|^{2}\right\rangle-\left\langle\left|E_{y}(\mathbf{q}, \omega, z)\right|^{2}\right\rangle-\left\langle\left|B_{x}(\mathbf{q}, \omega, z)\right|^{2}\right\rangle \\
& \left.-\left\langle\left|B_{y}(\mathbf{q}, \omega, z)\right|^{2}\right\rangle\right]_{z=0} .
\end{aligned}
$$

To linear order in the vibrational coordinate $u(t)$ and the frequency $\omega_{0}$, the stress acting on the surface 1 can be written in the form

$$
\sigma_{z z}=\sigma_{0 z z}(d)+u(t) \frac{\partial}{\partial d} \sigma_{0 z z}(d)+i \omega_{0} \gamma_{\perp} u(t) .
$$

Here the first term determines the conservative van der Waals stress and the second term is the adiabatic change of the conservative van der Waals stress during vibration. The last term determines the frictional stress with friction coefficient $\gamma_{\perp}$. For normal relative motion (see the Appendix) we ob- tain the friction coefficient $\gamma_{\perp}=\gamma_{\perp}^{\mathrm{rad}}+\gamma_{\perp}^{\text {evan }}$, where the contribution to the friction coefficient from the propagating electromagnetic waves is given by

$$
\begin{aligned}
\gamma_{\perp}^{\mathrm{rad}}= & \frac{\hbar}{4 \pi^{2}} \int_{0}^{\infty} d \omega\left(-\frac{\partial n}{\partial \omega}\right) \int_{0}^{\omega / c} d q q p^{2} \\
& \times\left[\left(1-\left|R_{1 p} R_{2 p}\right|^{2}\right)^{2}+\mid\left(1-\left|R_{1 p}\right|^{2}\right) R_{2 p} e^{i p d}\right. \\
& \left.+\left.\left(1-\left|R_{2 p}\right|^{2}\right) R_{1 p}^{*} e^{-i p d}\right|^{2}\right] \frac{1}{\left|1-e^{2 i p d} R_{1 p} R_{2 p}\right|^{4}} \\
& +[p \rightarrow s],
\end{aligned}
$$

and where the contribution to the friction from the evanescent electromagnetic waves is given by

$$
\begin{aligned}
\gamma_{\perp}^{\text {evan }}= & \frac{\hbar^{2}}{\pi^{2}} \int_{\omega / c}^{\infty} d \omega\left(-\frac{\partial n}{\partial \omega}\right) \int_{\omega / c}^{\infty} d q q k^{2} e^{-2 k d} \\
& \times\left[\left(\operatorname{Im} R_{1 p}+e^{-2 k d}\left|R_{1 p}\right|^{2} \operatorname{Im} R_{2 p}\right)\right. \\
& \times\left(\operatorname{Im} R_{2 p}+e^{-2 k d}\left|R_{2 p}\right|^{2} \operatorname{Im} R_{1 p}\right) \\
& \left.+e^{-2 k d}\left[\operatorname{Im}\left(R_{1 p} R_{2 p}\right)\right]^{2}\right] \frac{1}{\left|1-e^{-2 k d} R_{1 p} R_{2 p}\right|^{4}} \\
& +[p \rightarrow s],
\end{aligned}
$$

where $k=|p|$. The symbol $[p \rightarrow s]$ in Eqs. (22) and (23) denotes the term which is obtained from the first one by replacement of the reflection amplitude $R_{p}(\omega)$, for $p$-polarized waves, by the reflection amplitude $R_{S}(\omega)$ for $s$-polarized waves. The friction coefficient for two flat surfaces in parallel relative motion was obtained by us before, ${ }^{15}$ and can be written as $\gamma_{\|}=\gamma_{\|}^{\mathrm{rad}}+\gamma_{\|}^{\text {evan }}$, where the contribution to the friction coefficient from the propagating electromagnetic waves is given by

$$
\begin{aligned}
\gamma_{\|}^{\mathrm{rad}}= & \frac{\hbar}{8 \pi^{2}} \int_{0}^{\infty} d \omega\left(-\frac{\partial n}{\partial \omega}\right) \int_{0}^{\omega / c} d q q^{3} \\
& \times \frac{\left(1-\left|R_{1 p}\right|^{2}\right)\left(1-\left|R_{2 p}\right|^{2}\right)}{\left|1-e^{2 i p d} R_{1 p} R_{2 p}\right|^{2}}+[p \rightarrow s],
\end{aligned}
$$

and where the contribution to the friction from the evanescent electromagnetic waves is given by

$$
\begin{aligned}
\gamma_{\|}^{\mathrm{rad}}= & \frac{\hbar}{2 \pi^{2}} \int_{0}^{\infty} d \omega\left(-\frac{\partial n}{\partial \omega}\right) \int_{\omega / c}^{\infty} d q q^{3} e^{-2 k d} \\
& \times \operatorname{Im} R_{1 p} \operatorname{Im} R_{2 p} \frac{1}{\left|1-e^{-2 k d} R_{1 p} R_{2 p}\right|^{2}}+[p \rightarrow s] .
\end{aligned}
$$

There is a principal difference between the friction coefficient for normal and parallel relative motion, related to the denominator in the formulas for the friction coefficient. The resonant condition corresponds to the case when the denominator of the integrand in Eqs. (22)-(25), which is due to multiple scattering of the evanescent electromagnetic waves from the opposite surfaces, is small. For two identical surfaces and $R_{i} \ll 1 \leqslant R_{r}$, where $R_{i}$ and $R_{r}$ are the imaginary 
and real part of the reflection amplitude $\left(R=R_{r}+i R_{i}\right)$, this corresponds to the resonant condition $R_{r}^{2} \exp (-2 k d) \approx 1$. At resonance the denominators in the integrands of Eqs. (22)(25) have the small factors $R_{i}^{4}$ and $R_{i}^{2}$ for normal and parallel relative motion, respectively, and the nominators have the same factor $R_{i}^{2}$ in the both cases. Thus at resonance for normal relative motion the integrands in Eqs. (22) and (23) have a large addition factor $\sim 1 / R_{i}^{2}$, in comparison to the case of parallel relative motion. The resonance condition can be fulfilled even for the case when $\exp (-2 k d) \ll 1$ because for evanescent electromagnetic waves there is no restriction on the magnitude of the real part or the modulus of $R$. This open up the possibility of resonant denominators for $R_{r}^{2} \gg 1$.

\section{CASE OF THE GOOD CONDUCTORS}

A well-conducting metal has a dielectric function $\epsilon=1$ $-4 \pi i \sigma / \omega$ ( $\sigma$ is the conductivity) with an absolute value much larger than unity at thermal frequencies, and consequently $R_{p i} \ll 1$ and $R_{p r} \approx 1$. Thus an enhancement in friction due to multiple scattering of the electromagnetic waves from the opposite surfaces is possible only for very small $q$ $\ll 1 / d$. The entire subsequent calculation of the friction in this section is accurate to the leading order in the surface impedance $\zeta=\epsilon^{-1 / 2}=\zeta^{\prime}-i \zeta^{\prime \prime},|\zeta| \ll 1$.

It is convenient to write the friction coefficient for the two flat surfaces in the form

$$
\gamma=\hbar \int_{0}^{\infty} d \omega\left(-\frac{\partial n}{\partial \omega}\right)\left(I_{p}+I_{s}\right)
$$

Within the local optic approximation the reflection factors for the $s$ - and $p$-polarized electromagnetic waves are determined by the Fresnel formulas

$$
R_{p}=\frac{p-s / \epsilon}{p+s / \epsilon}, \quad R_{s}=\frac{p-s}{p+s}
$$

where

$$
s=\left[\left(\frac{\omega}{c}\right)^{2} \epsilon-q^{2}\right]^{1 / 2} .
$$

Taking into account that $q d q=k d k$, from Eq. (23) for normal relative motion of clean surfaces within the local optic approximation, we get the following contribution to the friction from the evanescent $p$-and $s$-polarized electromagnetic waves:

$$
\begin{aligned}
I_{\perp p}^{\mathrm{evan}}= & \int_{0}^{\infty} \frac{d k}{\pi^{2}} k^{5}[\operatorname{Re}(s / \epsilon)]^{2}\left[\left[\left(k^{2}+|s / \epsilon|^{2}\right) \cosh k d\right.\right. \\
& \left.+2 k[\operatorname{Im}(s / \epsilon)] \sinh k d]^{2}+\left(k^{2}-|s / \epsilon|^{2}\right)^{2}\right] \\
& \times \frac{1}{\left|\left((s / \epsilon)^{2}-k^{2}\right) \sinh k d+2 i k(s / \epsilon) \cosh k d\right|^{4}}
\end{aligned}
$$

$$
\begin{aligned}
I_{\perp s}^{\mathrm{evan}}= & \int_{0}^{\infty} \frac{d k}{\pi^{2}} k^{5}[\operatorname{Re} s]^{2}\left[\left[\left(k^{2}+|s|^{2}\right) \cosh k d\right.\right. \\
& \left.+2 k \operatorname{Im} s \sinh k d]^{2}+\left(k^{2}-|s|^{2}\right)^{2}\right] \\
& \times \frac{1}{\left|\left(s^{2}-k^{2}\right) \sinh k d+2 i k s \cosh k d\right|^{4}} .
\end{aligned}
$$

For $I_{\perp p}^{\text {evan }}$, for $(c / \omega)|\epsilon|^{-3 / 2}<d<(c / \omega)|\epsilon|^{1 / 2}$ and $|\zeta| \ll 1$ there is a singularity in the integrand of the type $-1 / k^{3}$ in the limit $k \rightarrow 0$, and the main contribution to the integral comes from this singularity. Taking into account that $\sinh k d \approx k d$ and $\cosh k d \approx 1$ as $k \rightarrow 0$, to first order in $\zeta$ we get

$$
\begin{aligned}
I_{\perp p}^{\mathrm{evan}} & =2(\omega / c)^{2} \zeta^{\prime} \int_{0}^{\infty} \frac{d k}{\pi^{2}} \frac{k^{5}}{\left|k^{2} d-2 i(\omega / c)(\zeta)\right|^{4}} \\
& =\frac{\omega \zeta^{\prime}}{\pi^{2} c d^{3}}\left(\frac{\pi}{2}+\arctan \zeta^{\prime \prime} / \zeta^{\prime}-\frac{\zeta^{\prime \prime} / \zeta^{\prime}}{1+\left(\zeta^{\prime \prime} / \zeta^{\prime}\right)^{2}}\right) .
\end{aligned}
$$

As $k \rightarrow 0$, there is no singularity in the integrand of $I_{\perp s}^{\text {evan }}$, thus the main contribution to the integral comes from $k$ $\sim d^{-1}$. For $d<(c / \omega)|\epsilon|^{-1 / 2}, I_{s}$ becomes slowly dependent on $d$ :

$$
\begin{aligned}
I_{\perp s} & \approx \int_{0}^{\infty} \frac{d k}{\pi^{2}} k\left[\sqrt{k^{4}+(\omega / c)^{4}|\epsilon|^{2}}-k^{2}\right] e^{-2 k d} \\
& \approx \frac{1}{8 \pi^{2}}(\omega / c)^{4}|\epsilon|^{2}\left(1.22-\ln \left(2 d|\epsilon|^{1 / 2} \omega / c\right)\right),
\end{aligned}
$$

while for $d>(c / \omega)|\epsilon|^{-1 / 2}$ we get

$$
I_{\perp s} \approx(c / \omega)^{2} \zeta^{\prime 2} d^{-6} .
$$

For the propagating electromagnetic waves, taking into account that $q d q=-p d p$, we get

$$
I_{\perp p}^{\mathrm{rad}}=(\omega / c)^{2} \zeta^{\prime 2} \int_{0}^{\omega / c} \frac{d p}{\pi^{2}} p^{5} \frac{1+\cos ^{2}(p d)}{|p \sin p d+2 i(\omega / c) \zeta \cos p d|^{4}},
$$

$$
I_{\perp s}^{\mathrm{rad}}=(\omega / c)^{2} \zeta^{\prime 2} \int_{0}^{\omega / c} \frac{d p}{\pi^{2}} p^{5} \frac{1+\cos ^{2}(p d)}{|(\omega / c) \sin p d+2 i p \zeta \cos p d|^{4}} .
$$

For $d<(c / \omega)|\epsilon|^{-1 / 2}$ the contribution to the friction from the propagating wave is negligibly small in the comparison with the contribution from the evanescent waves. For $d$ $>(c / \omega)|\epsilon|^{-1 / 2}$ the main contribution to integrals (34) and (35) comes from the integration near the singularities at $p$ $=p_{n}=\pi n / d<\omega / c$ (where $n$ is an integer), when $\sin k d=0$. For the contribution to $I_{p}^{\mathrm{rad}}$ from singularity at $n=0$ we get

$$
I_{\perp p}^{\mathrm{rad}} \approx \frac{\omega \zeta^{\prime}}{4 \pi^{2} c d^{3}}\left(\frac{\pi}{2}-\arctan \zeta^{\prime \prime} / \zeta^{\prime}+\frac{\zeta^{\prime \prime} / \zeta^{\prime}}{1+\left(\zeta^{\prime \prime} / \zeta^{\prime}\right)^{2}}\right) .
$$

In the vicinity of other singularities $p_{n} \neq 0$, putting $p=p_{n}$ $+p^{\prime}$, we have $\sin p d \approx(-1)^{n} p^{\prime} d$ and $\cos p d \approx(-1)^{n}$, 


$$
\begin{aligned}
I_{\perp p}^{\mathrm{rad}} & \approx 2(\omega / c)^{2} \zeta^{\prime 2} \int \frac{d p^{\prime}}{\pi^{2}} \frac{1}{\left|p_{n} p^{\prime} d+2 i(\omega / c) \zeta\right|^{4}} \\
& \approx \frac{p_{n}^{4} c}{8 \pi^{2} \omega d \zeta^{\prime}}\left(\frac{\pi}{2}-\arctan \zeta^{\prime \prime} / \zeta^{\prime}-\frac{\zeta^{\prime \prime} / \zeta^{\prime}}{1+\left(\zeta^{\prime \prime} / \zeta^{\prime}\right)^{2}}\right) .
\end{aligned}
$$

The number $m$ of such contribution is obviously equal to the integer part of the quantity $y=\omega d / \pi c(m=[y])$, so that the total $p_{n} \neq 0$ contribution becomes

$$
\begin{aligned}
\frac{\pi^{2} c}{8 \omega d^{5} \zeta^{\prime}} & \left(\frac{\pi}{2}-\arctan \zeta^{\prime \prime} / \zeta^{\prime}-\frac{\zeta^{\prime \prime} / \zeta^{\prime}}{1+\left(\zeta^{\prime \prime} / \zeta^{\prime}\right)^{2}}\right) \sum_{n=1}^{m} n^{4} \\
= & \frac{\pi^{2} c}{8 \omega d^{5} \zeta^{\prime}}\left(\frac{\pi}{2}-\arctan \zeta^{\prime \prime} / \zeta^{\prime}-\frac{\zeta^{\prime \prime} / \zeta^{\prime}}{1+\left(\zeta^{\prime \prime} / \zeta^{\prime}\right)^{2}}\right) \\
& \times\left[\frac{(m+1)^{5}}{5}-\frac{(m+1)^{4}}{2}+\frac{(m+1)^{3}}{3}-\frac{m}{30}-\frac{1}{30}\right] .
\end{aligned}
$$

In the integral $I_{\perp s}^{\mathrm{rad}}$ there is no singularity at $p_{n}=0$, and the contribution from the vicinity of the point $p_{n} \neq 0$ is

$$
\begin{aligned}
& 2(\omega / c)^{2} \zeta^{\prime 2} \int_{0}^{\omega / c} \frac{d p^{\prime}}{\pi^{2}} \frac{p_{n}^{5}}{\left|(\omega / c) p^{\prime} d+2 i p_{n} \zeta\right|^{4}} \\
& =\frac{\omega n^{2}}{8 c d^{3} \zeta^{\prime}}\left(\frac{\pi}{2}-\arctan \zeta^{\prime \prime} / \zeta^{\prime}-\frac{\zeta^{\prime \prime} / \zeta^{\prime}}{1+\left(\zeta^{\prime \prime} / \zeta^{\prime}\right)^{2}}\right),
\end{aligned}
$$

and, consequently,

$$
\begin{aligned}
I_{\perp s}^{\mathrm{rad}}= & \frac{\omega}{8 c d^{3} \zeta^{\prime}}\left(\frac{\pi}{2}-\arctan \zeta^{\prime \prime} / \zeta^{\prime}-\frac{\zeta^{\prime \prime} / \zeta^{\prime}}{1+\left(\zeta^{\prime \prime} / \zeta^{\prime}\right)^{2}}\right) \sum_{n=1}^{m} n^{2} \\
= & \frac{\omega}{48 c d^{3} \zeta^{\prime}}\left(\frac{\pi}{2}-\arctan \zeta^{\prime \prime} / \zeta^{\prime}-\frac{\zeta^{\prime \prime} / \zeta^{\prime}}{1+\left(\zeta^{\prime \prime} / \zeta^{\prime}\right)^{2}}\right) \\
& \times m(m+1)(2 m+1) .
\end{aligned}
$$

For $m \gg 1$, where we can assume $m \approx \pi \omega / c d$, the $s$ - and $p$-wave contributions are approximately equal, and for the total contribution from propagating electromagnetic waves in this limit we get

$$
I_{\perp}^{\mathrm{rad}}=I_{\perp p}^{\mathrm{rad}}+I_{\perp s}^{\mathrm{rad}} \approx \frac{11 \omega^{4}}{240 \pi^{3} c^{4} \zeta^{\prime}} .
$$

The above formulas were obtained from the Eqs. (22) and (23) by neglecting the spatial dispersion of the dielectric function. But these formulas depend only on the solids via the surface impedance $\zeta$, which is equal to the ratio of the tangential components of the electric and magnetic fields on the boundary of the body. Thus, the results in this section also remain valid in the presence of spatial dispersion, provided only that the surface impedance of the medium is small enough. Thus, we would have arrived at the same formulas if we had assumed from the very beginning that the Leontovich boundary condition $\mathbf{E}=\boldsymbol{\zeta} \mathbf{H} \times \mathbf{n}$ is satisfied on the surface of the metal.
At not too low temperatures, the impedances of metals are given by

$$
\zeta^{\prime}=\zeta^{\prime \prime}=(\omega / 8 \pi \sigma)^{1 / 2}
$$

In the local optic approximation we assume that there is no dependence of $\sigma$ on q. In the Wien region of frequencies it is also a good approximation to neglect the frequency dependence of $\sigma$. In this approximation using Eq. (31) for $\lambda_{W}\left(k_{B} T / 4 \pi \hbar \sigma\right)^{3 / 2}<d<\lambda_{W}\left(4 \pi \hbar \sigma /\left(k_{B} T\right)^{1 / 2}\right) \quad\left(\lambda_{W}\right.$ $\left.=c \hbar /\left(k_{B} T\right)\right)$, we get

$$
\gamma_{\perp p}^{\mathrm{evan}}=\hbar \int_{0}^{\infty} d \omega\left(-\frac{\partial n}{\partial \omega}\right) I_{\perp p}^{\mathrm{evan}} \approx 0.13 \frac{\hbar}{d^{3} \lambda_{W}}\left(\frac{k_{B} T}{4 \pi \hbar \sigma}\right)^{1 / 2}
$$

For comparison, the $p$-wave contribution for parallel relative motion for $d<\lambda_{c},\left(\lambda_{c}=c /\left(4 \pi \sigma k_{B} T\right)^{1 / 2}\right)$ is given by ${ }^{15,17}$

$$
\gamma_{\| p}^{\text {evan }} \approx 0.3 \frac{\hbar}{d^{4}}\left(\frac{k_{B} T}{4 \pi \hbar \sigma}\right)^{2}
$$

It is interesting to note that for normal relative motion, in contrast to parallel relative motion, practically for all $d>0$ the main contribution to friction comes from retardation effects, since Eq. (42), in contrast to Eq. (43), contains the light velocity.

From Eq. (32) we get the $s$-wave contribution to friction for $d<\lambda_{c}$ :

$$
\gamma_{\perp s}^{\text {evan }} \approx 10^{-2} \frac{\hbar}{\lambda_{c}^{4}}\left[3-5 \ln \left(2 d / \lambda_{c}\right)\right] .
$$

For parallel relative motion the $s$-wave contribution is two times smaller.

For $d>\lambda_{c}$, taking into account that Eq. (33) is valid only for $\omega>c^{2} / 4 \pi \sigma d^{2}$, we get

$$
\gamma_{\perp s}^{\mathrm{evan}} \approx \frac{\pi k_{B} T \sigma}{d^{2} c^{2}}
$$

From Eq. (40) for $d>\lambda_{W}$ we get a distance independent contribution to the friction from propagating electromagnetic waves

$$
\gamma_{\perp}^{\mathrm{rad}} \approx 1.9 \cdot 10^{-2} \frac{\hbar}{\lambda_{W}^{3} \lambda_{c}}
$$

\section{PHOTON TUNNELING ENHANCEMENT OF THE VAN DER WAALS FRICTION}

We rewrite the denominator of Eq. (23) in the form

$$
\begin{aligned}
\left|1-e^{-2 k d} R^{2}\right|^{4}= & {\left[\left(1-e^{-k d} R_{r}\right)^{2}+e^{-2 k d} R_{i}^{2}\right]^{2} } \\
& \times\left[\left(1+e^{-k d} R_{r}\right)^{2}+e^{-2 k d} R_{i}^{2}\right]^{2},
\end{aligned}
$$

where $R_{r}$ and $R_{i}$ are real and imaginary parts of $R$, respectively $\left(R=R_{r}+i R_{i}\right)$. Let us suppose that $\left|R_{r}\right| \gg R_{i}$. In this case resonant conditions are determined by the equation

$$
R_{r}\left(\omega_{ \pm}(k)\right)= \pm e^{k d}
$$


Close to resonance we can write

$$
\begin{aligned}
{\left[\left(1 \pm e^{-k d} R_{r}\right)^{2}+e^{-2 k d} R_{i}^{2}\right] \approx } & e^{-2 k d} R_{r}^{\prime 2}\left(\omega_{ \pm}\right)\left\{\left(\omega-\omega_{ \pm}\right)^{2}\right. \\
& \left.+\left[R_{i}\left(\omega_{ \pm}\right) / R_{r}^{\prime}\left(\omega_{ \pm}\right)\right]^{2}\right\},
\end{aligned}
$$

where

$$
R_{r}^{\prime}\left(\omega_{ \pm}\right)=\left.\frac{d R_{r}(\omega)}{d \omega}\right|_{\omega=\omega_{ \pm}},
$$

which leads to the following contribution to the friction coefficient:

$$
\gamma_{\perp \pm} \approx \frac{\hbar^{2}}{16 k_{B} T} \int_{0}^{q_{c}} d k k^{3} \frac{e^{2 k d}}{\left[\left|R_{r}^{\prime}\left(\omega_{ \pm}\right)\right| R_{i}\left(\omega_{ \pm}\right) \sinh ^{2} \hbar \omega_{ \pm} / 2 k_{B} T\right]} .
$$

The parameter $q_{c}$ in this expression defines the region 0 $<k<q_{c}$ where the two pole approximation is valid. To proceed further let us make the following simplifications. Close to a pole we can use the approximation

$$
R=\frac{a}{\omega-\omega_{0}-i \eta},
$$

where $a$ is a constant. Then from resonant condition (48) we get

$$
\omega_{ \pm}=\omega_{0} \pm a e^{-k d} .
$$

For the two poles approximation to be valid the difference $\Delta \omega=\left|\omega_{+}-\omega_{-}\right|$must be greater than the width $\eta$ of the resonance. From this condition we get $q_{c} \leqslant \ln (2 a / \eta) / d$. For short distances the parameter $q_{c}$ defines the value of $k$ where the solution of Eq. (48) ceases to exit.

For $\omega_{0}>a$ and $q_{c} d>1$, from Eq. (50) we get

$$
\gamma_{\perp \pm}=\frac{3}{128} \frac{\hbar^{2} a^{2}}{d^{4} k_{B} T \eta} \frac{1}{\sinh ^{2}\left(\hbar \omega_{0} / 2 k_{B} T\right)} .
$$

For parallel relative motion, using the same approximation as above, we get

$$
\gamma_{\|}=\frac{\hbar^{2} \eta q_{c}^{4}}{128 \pi k_{B} T} \frac{1}{\sinh ^{2}\left(\hbar \omega_{0} / 2 k_{B} T\right)} .
$$

Interestingly, the explicit $d$ dependence has dropped out of Eq. (53). However, $\gamma_{\|}$is still $d$ dependent, due to the $d$ dependence of $q_{c}$. For small distances one can expect that $q_{c}$ is determined by the dielectric properties of the material and does not depend on $d$. In this case the friction will also be distance independent. Thus, perhaps the weak distance dependence observed in Ref. 7 can be explained by the resonant photon tunneling.

\section{NUMERICAL RESULTS}

At $d<l, v_{F} / \omega$, where $l$ is the electron mean free path, and where $v_{F}$ is the Fermi velocity, respectively, the system will be characterized by a nonlocal dielectric function $\epsilon(\mathbf{q}, \omega)$. In this paper we use the nonlocal optic dielectric approach, proposed some years ago for investigations of the optical properties of a semi-infinite electron gas, ${ }^{20}$ which should accurately describe the nonlocal optic effects.

Accordingly to Ref. 20, the reflection factor for a $p$-polarized electromagnetic field, incident on the flat surface, is determined by ${ }^{20}$

$$
R_{p}=\frac{p-Z_{p}}{q+Z_{p}},
$$

where the surface impedance $Z_{p}$ is given by

$$
Z_{p}=\frac{2 i}{\pi} \int_{0}^{\infty} \frac{d q_{z}}{Q^{2}}\left(\frac{q^{2}}{\epsilon_{l}(\omega, Q)}+\frac{(\omega / c)^{2} q_{z}^{2}}{(\omega / c)^{2} \epsilon_{t}(\omega, Q)-Q^{2}}\right),
$$

where $\epsilon_{l}$ is the finite lifetime generalization of the longitudinal Lindhard dielectric function, which, according to Ref. 21, can be written as

$$
\begin{gathered}
\epsilon_{l}(\omega, \Omega)=1 \\
+\frac{(1+i / \omega \tau)\left[\epsilon_{l}^{0}(\omega+i / \tau, Q)-1\right]}{1+(i / \omega \tau)\left[\epsilon_{l}^{0}(\omega+i / \tau, Q)-1\right] /\left[\epsilon_{l}^{0}(0, Q)-1\right]} \\
\quad \epsilon_{l}^{0}(\omega, Q)=1+\frac{3 \omega_{p}^{2}}{Q^{2} v_{F}^{2}} f_{l} \\
f_{l}=\frac{1}{2}+\frac{1}{8 z}\left(\left[1-(z-u)^{2}\right] \ln \frac{z-u+1}{z-u-1}\right. \\
\left.+\left[1-(z+u)^{2}\right] \ln \frac{z+u+1}{z+u-1}\right)
\end{gathered}
$$

where $Q^{2}=q^{2}+q_{z}^{2}, \quad z=Q / 2 k_{F}, \quad u=\omega /\left(Q v_{F}\right), \quad \omega_{p}$ is the plasma frequency, $\tau$ is the Drude relaxation time, where $v_{F}$ and $k_{F}$ are the Fermi velocity and wave vector, respectively. For $s$ polarization the reflection factor is determined by

$$
R_{s}=\frac{1-Z_{s} p}{1+Z_{s} p},
$$

where

$$
\begin{gathered}
Z_{s}=\frac{2 i}{\pi} \int_{0}^{\infty} \frac{d q_{z}}{(\omega / c)^{2} \epsilon_{t}(\omega, Q)-Q^{2}} \\
\epsilon_{t}(\omega, Q)=1-\frac{\omega_{p}^{2}}{\omega(\omega+i \gamma)} f_{t} \\
f_{t}=\frac{3}{8}\left(z^{2}+3 u^{\prime 2}+1\right)-\frac{3}{32 z}\left(\left[1-\left(z-u^{\prime}\right)^{2}\right]^{2} \ln \frac{z-u^{\prime}+1}{z-u^{\prime}-1}\right. \\
\left.+\left[1-\left(z+u^{\prime}\right)^{2}\right]^{2} \ln \frac{z+u^{\prime}+1}{z+u^{\prime}-1}\right)
\end{gathered}
$$

with $u^{\prime}=\left(\omega+i \tau^{-1}\right) /\left(Q v_{F}\right)$. We will show below that the maximum of the van der Waals friction is reached for small 


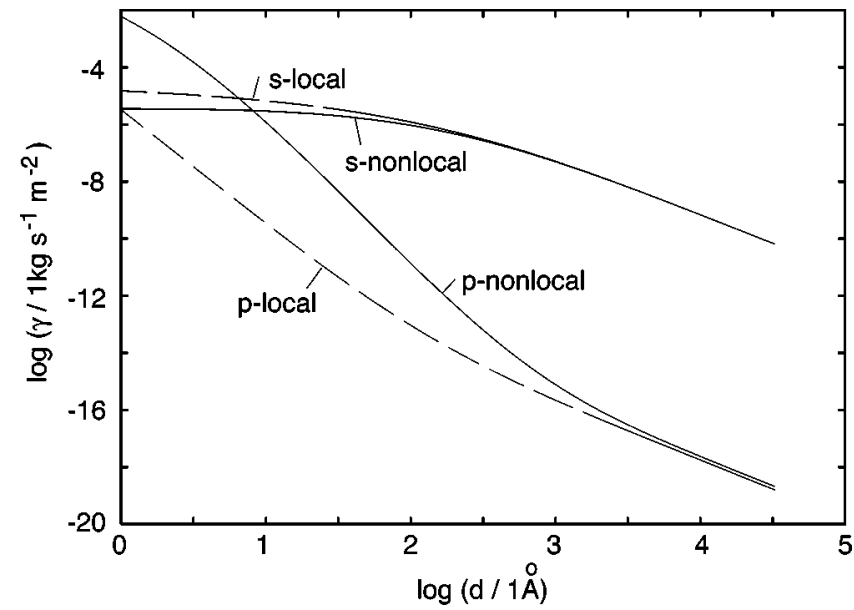

FIG. 1. The friction coefficient for two flat surfaces in parallel relative motion as a function of separation $d$ at $T=273 \mathrm{~K}$ with parameter chosen to correspond to copper $\left(\tau^{-1}=2.5 \times 10^{13} \mathrm{~s}^{-1}\right.$ and $\left.\omega_{p}=1.6 \times 10^{16} \mathrm{~s}^{-1}\right)$. The contributions from the $s$ - and $p$-polarized electromagnetic fields are shown separately. The full curves represent the results obtained within the nonlocal optic dielectric formalism, and the dashed curves represent the result obtained within the local optic approximation. (The log function is with basis 10.)

electron densities, where the electron gas becomes nondegenerate (the electron gas is degenerate for $k_{B} T \ll \varepsilon_{F}$ and nondegenerate for $k_{B} T \geqslant \varepsilon_{F}$, where $\varepsilon_{F}$ is the Fermi energy). For a nondegenerate electron gas we use the following classical expressions for dielectric functions: ${ }^{22}$

$$
\begin{gathered}
\epsilon_{l}^{0}(\omega, Q)=1+\left(\frac{\omega_{p}}{Q v_{T}}\right)^{2}\left[1+F\left(\frac{\omega}{\sqrt{2} Q v_{T}}\right)\right], \\
\epsilon_{t}(\omega, Q)=1+\frac{\omega_{p}^{2}}{\omega(\omega+i \gamma)} F\left(\frac{\omega+i \gamma}{\sqrt{2} Q v_{T}}\right),
\end{gathered}
$$

where the function $F(x)$ is defined by the integral

$$
F(x)=\frac{x}{\sqrt{\pi}} \int_{-\infty}^{+\infty} d z \frac{e^{-z^{2}}}{z-x-i 0},
$$

and $v_{T}=\sqrt{k_{B} T / m}$, where $m$ is the electron mass.

Figures 1 and 2 show the calculated contribution to the friction coefficient $\gamma$ from evanescent electromagnetic waves for two semi-infinite solids, with parameters chosen to correspond to copper $\left(\tau^{-1}=2.5 \times 10^{13} \mathrm{~s}^{-1}\right.$ and $\omega_{p}=1.6$ $\times 10^{16} \mathrm{~s}^{-1}$ ) at $T=273 \mathrm{~K}$, for parallel (Fig. 1) and normal (Fig. 2) relative motions. Results are shown separately for both the $s$ - and $p$-wave contributions. The dashed line show the result when the local (long-wavelength) dielectric function $\epsilon(\omega)=\epsilon_{l}=\epsilon_{t}$ is used, where

$$
\epsilon(\omega)=1-\frac{\omega_{p}^{2}}{\omega\left(\omega+i \tau^{-1}\right)} .
$$

In this case the integration in Eqs. (55) and (60) can be performed analytically resulting in Fresnel formulas. Figure 1 shows that, for sufficiently small separations (d

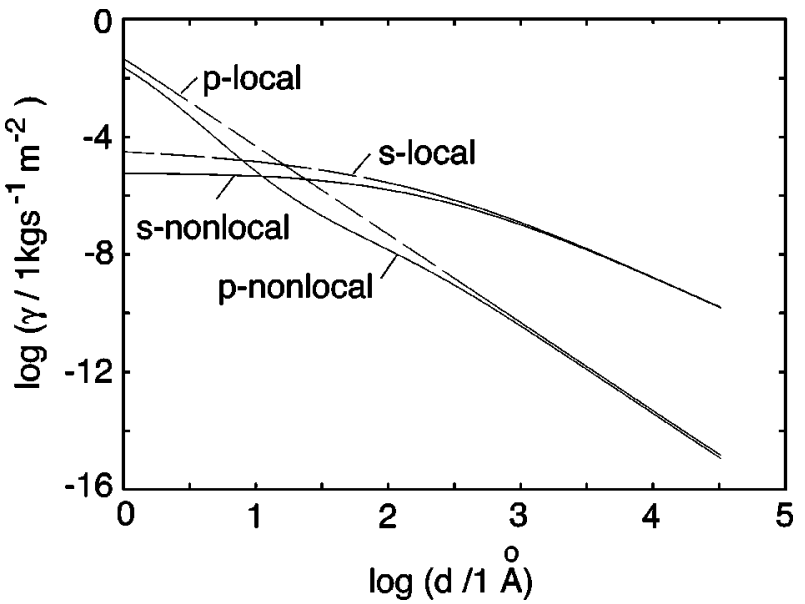

FIG. 2. The friction coefficient for two flat surfaces in normal relative motion as a function of separation $d$ at $T=273 \mathrm{~K}$ with parameter chosen to correspond to copper $\left(\tau^{-1}=2.5 \times 10^{13} \mathrm{~s}^{-1}\right.$ and $\left.\omega_{p}=1.6 \times 10^{16} \mathrm{~s}^{-1}\right)$. The contributions from the $s$ - and $p$-polarized electromagnetic fields are shown separately. The full curves represent the results obtained within the nonlocal optic dielectric formalism, and the dashed curves represent the result obtained within the local optic approximation. (The log function is with basis 10.)

$<1000 \AA$ ), for parallel relative motion the nonlocal optic effects become important for the $p$-wave contribution. However, for the $s$-wave contribution, for both parallel and normal relative motion, the nonlocal optic effects are negligibly small for practically all separations. For normal relative motion, for the $p$-wave contribution the nonlocal optic effects are less important than for the parallel relative motion. In the present calculations we have taken into account the nonlocal effects in the bulk of the solids. There are also nonlocal contributions from the surface region which we investigated in our previous publications. ${ }^{16,17}$ Comparing our previous calculations with the present one, we find that for $d>10 \AA$ the volume contribution from the nonlocal effects is of the same importance as the surface contribution.

To estimate the friction coefficient $\Gamma$ for an atomic force microscope tip we can use an approximate formula ${ }^{23,24}$

$$
\Gamma=2 \pi \int_{0}^{\infty} d \rho \rho \gamma[z(\rho)]
$$

where it is assumed that the tip has cylinder symmetry. Here $z(\rho)$ denotes the tip-surface distance as a function of the distance $\rho$ from the tip symmetry axis, and the friction coefficient $\gamma[z(\rho)]$ is determined by the expressions for the flat surfaces. This scheme was proposed in Ref. 23 for the calculation of the conservative van der Waals interaction. The error of these scheme is not larger than 5-10\% in practice in an atomic force microscopy experiment, and $25 \%$ in a worst case situation. ${ }^{24}$ Although this scheme was proposed for the conservative van der Waals interaction, we assume that the same scheme is also valid for the calculation of the van der Waals friction. We assume that the tip has a paraboloid shape given [in cylindrical coordinates $(z, \rho)$ ] by the formula: $z$ $=d+\rho^{2} / 2 R$, where $d$ is the distance between the tip and the 
flat surface, and where $R$ is the radius of curvature of the tip. In the case of the power dependence

$$
\gamma(\rho)=\frac{C}{\left(d+\frac{\rho^{2}}{2 R}\right)^{n}},
$$

we get

$$
\Gamma=\frac{2 \pi R}{n-1} \frac{C}{d^{n-1}}=\frac{2 \pi R d}{n-1} \gamma(d)=A_{\mathrm{eff}} \gamma(d),
$$

where $A_{\text {eff }}=2 \pi R d /(n-1)$ is the effective surface area. In a more general case one must use numerical integration.

For $d>10 \AA$ the main contribution to the friction coefficient $\gamma$ comes from $s$-polarized waves. In particularly, at $d$ $=100 \AA$ the $s$-wave contribution $\gamma_{s} \approx 10^{-5} \mathrm{kgs}^{-1} \mathrm{~m}^{-2}$, so that with the effective surface area $A_{\mathrm{eff}} \approx 10^{-14} \mathrm{~m}^{-2}$ (typical for probe scanning microscopy), the friction coefficient is $\Gamma \approx \gamma_{s} A_{\text {eff }} \sim 10^{-19} \mathrm{kgs}^{-1}$. The $s$-wave contribution is characterized by weak distance dependence for $d<100 \AA$, and $\gamma$ $\sim d^{-2}$ for $d>100 \AA$. For good conductors like copper, even for very short distances, the main contribution to the friction coefficient comes from the $s$-polarized electromagnetic waves. This difference between $p$ - and $s$-polarized waves results from screening effects: Good conductors are good reflectors for the $p$-polarized field, which implies that they are ineffective in the emission and absorption of evanescent $p$-polarized waves. However these screening effects are less important for $s$-polarized waves.

As pointed out in Refs. 4, 15, 17, and 18, the $p$-wave contribution increase and the $s$-wave contribution decrease when the free electron density decrease. Within the local optic approximation the friction diverges in the limit of zero conductivity. This situation is different from the radiative heat transfer, where, even in the local optics approximation, a maximum in the heat transfer occurs for conductivities corresponding to semimetals. Figure 3 shows the dependence of the coefficient of friction on the electron density. When the electron density decreases there is transition from a degenerate electron gas to a nondegenerate electron gas at the density $n_{F} \sim\left(k_{B} T m\right)^{3 / 2} / \pi^{2} \hbar^{3}$. At $T=273 \mathrm{~K}$ the transition density is $n_{F} \sim 10^{25} \mathrm{~m}^{-3}$. For $n>n_{F}$ we use the (nonlocal) dielectric function appropriate for a degenerate electron gas, while for $n<n_{F}$ we use an expression corresponding to a nondegenerate electron gas. In the calculations we used the electron mean free path $l \approx 600 \AA$. At $d=100 \AA$ the maximum value $\gamma_{\max } \sim 10^{-4} \mathrm{~kg} \mathrm{~s}^{-1}$ is obtained for $n_{\max }$ $\sim 10^{22} \mathrm{~m}^{-3}$, corresponding to the dc conductivity $\sigma$ $\sim 1(\Omega \mathrm{m})^{-1}$.

Resonant photon tunneling enhancement of the van der Waals friction is possible for two semiconductor surfaces which can support low-frequency surface plasmon modes. As an example we consider two clean surfaces of silicon carbide $(\mathrm{SiC})$. The optical properties of this material can be described using an oscillator model ${ }^{25}$

$$
\epsilon(\omega)=\epsilon_{\infty}\left(1+\frac{\omega_{L}^{2}-\omega_{T}^{2}}{\omega_{T}^{2}-\omega^{2}-i \Gamma \omega}\right),
$$

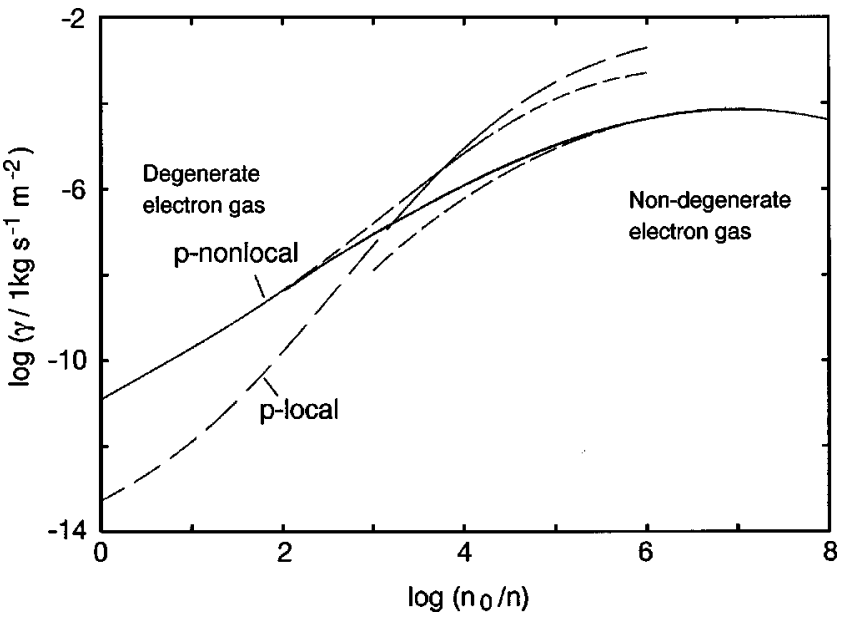

FIG. 3. The friction coefficient for two flat surfaces in parallel relative motion as a function of the free electron density $n$ at $T$ $=273 \mathrm{~K}$. The full curve was obtained by interpolation between the result (dashed lines) obtained within the nonlocal optic dielectric approach, with dielectric functions corresponding to a degenerate electron gas for $n>n_{F} \sim 10^{25} \mathrm{~m}^{-3}$, and to a nondegenerate electron gas for $n<n_{F}$. The calculation were performed with the damping constant $\tau^{-1}=2.5 \times 10^{13} \mathrm{~s}^{-1}$, separation $d=100 \AA$, and $n_{0}=8.6$ $\times 10^{28} \mathrm{~m}^{-3}$. (The log function is with basis 10 .)

with $\epsilon_{\infty}=6.7, \omega_{L}=1.8 \times 10^{14} \mathrm{~s}^{-1}, \omega_{T}=1.49 \times 10^{14} \mathrm{~s}^{-1}$, and $\Gamma=8.9 \times 10^{11} \mathrm{~s}^{-1}$. The frequency of surface plasmons is determined by condition $\epsilon_{r}\left(\omega_{p}\right)=-1$ and from Eq. (6) we get $\omega_{p}=1.78 \times 10^{14} \mathrm{~s}^{-1}$. In Fig. 4 we plot the friction coefficient $\gamma(d)$ : note that the friction between the two semiconductor surfaces is several order of magnitude larger than between two clean good conductor surfaces.

Another enhancement mechanism is connected with resonant photon tunneling between adsorbate vibrational modes localized on different surfaces. In the local optic approximation, where the dielectric function is assumed to depend only on the frequency $\omega$, the reflection factors $R_{p}$ and $R_{s}$ for flat surfaces, covered by an adsorbate layer, are given by: ${ }^{26}$

$$
R_{p}=\frac{p-s / \epsilon-4 \pi i n_{a} q\left[s \alpha_{\|} / \epsilon-q \alpha_{\perp}\right]}{p+s / \epsilon-4 \pi i n_{a} q\left[s \alpha_{\|} / \epsilon+q \alpha_{\perp}\right]},
$$

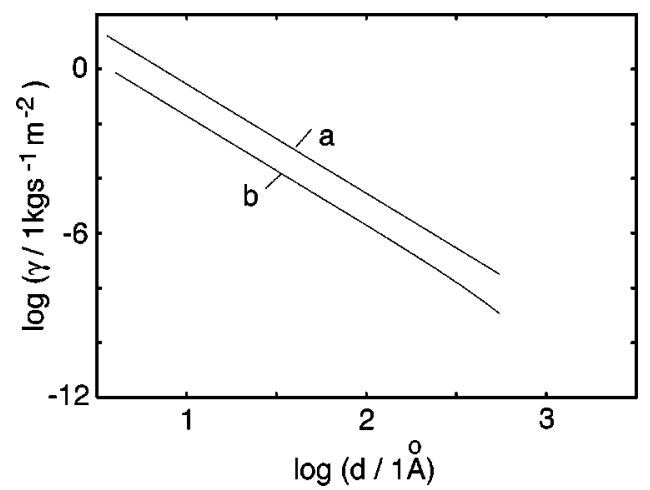

FIG. 4. The friction coefficient for two clean semiconductor surfaces in (a) normal and (b) parallel relative motions, as a function of the separation $d . T=300 \mathrm{~K}$ and with parameters chosen to correspond to a surfaces of silicon carbide $(\mathrm{SiC})$ (see the text for an explanation). (The log-function is with basis 10.) 


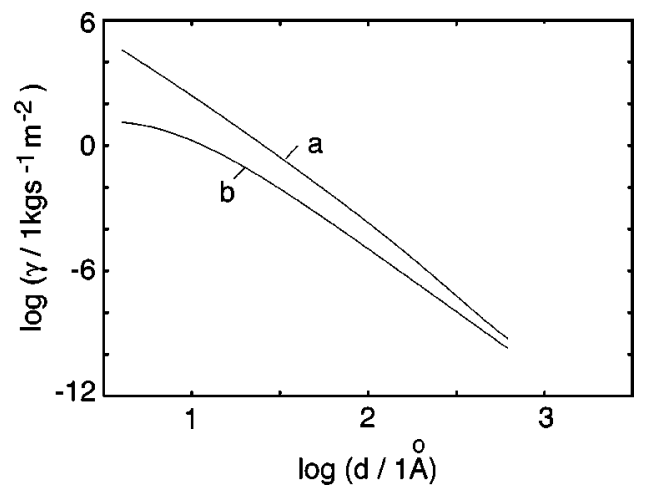

FIG. 5. The friction coefficient for two surface covered by adsorbates in (a) normal and (b) parallel relative motion, as a function of the separation $d . T=273 \mathrm{~K}$ and with parameters chosen to correspond to $\mathrm{K} / \mathrm{Cu}(001)$ (Ref. 28). $\left(\omega_{\perp}=1.9 \times 10^{13} \mathrm{~s}^{-1}, \omega_{\|}=4.5\right.$ $\times 10^{12} \mathrm{~s}^{-1}, \quad \eta_{\|}=2.8 \times 10^{10} \mathrm{~s}^{-1}, \quad \eta_{\perp}=1.6 \times 10^{12} \mathrm{~s}^{-1}, \quad$ and $e^{*}$ $=0.88 e$ ). (The log function is with basis 10.)

$$
R_{s}=\frac{p-s-4 \pi i n_{a}(\omega / c)^{2} \alpha_{\|}}{p+s+4 \pi i n_{a}(\omega / c)^{2} \alpha_{\|}},
$$

and where $\alpha_{\|}$and $\alpha_{\perp}$ are the polarizabilities of adsorbates in a direction parallel and normal to the surface, respectively. Here $\epsilon=\epsilon(\omega)$ is the bulk dielectric function and $n_{a}$ is the concentration of adsorbates. For clean surfaces $n_{a}=0$, and in this case formulas (70) and (71) reduce to the well-known Fresnel formulas.

As an example, let us consider ions with charge $e^{*}$ adsorbed on metal surfaces. The polarizability for ion vibration normal to the surface is given by

$$
\alpha_{\perp}=\frac{e^{* 2}}{M\left(\omega_{\perp}^{2}-\omega^{2}-i \omega \eta_{\perp}\right)},
$$

where $\omega_{\perp}$ is the frequency of the normal adsorbate vibration, and $\eta_{\perp}$ is the damping constant. In Eq. (70) the contribution from parallel vibrations is reduced by the small factor $1 / \epsilon$. However, the contribution of parallel vibrations to the van der Waals friction can nevertheless be important due to the indirect interaction of parallel adsorbate vibration with the electric field, via the metal conduction electron. ${ }^{27}$ Thus the small parallel component of the electric field will induce a strong electric current in the metal. The drag force between the electron flow and adsorbates can induce adsorbate vibrations parallel to the surface. This gives the polarizability

$$
\alpha_{\|}=\frac{\epsilon-1}{n} \frac{e^{*}}{e} \frac{\omega \eta_{\|}}{\left(\omega_{\|}^{2}-\omega^{2}-i \omega \eta_{\|}\right)},
$$

where $n$ is the conduction electron concentration. As an illustration, in Fig. 5 we show coefficient of friction for the two $\mathrm{Cu}(001)$ surfaces covered by a low concentration of potassium atoms $\left(n_{a}=10^{18} \mathrm{~m}^{-2}\right)$. In the $q$ integral in Eqs. (23) and (25) we used the cutoff $q_{c} \sim \pi / a$ (where $a \approx 1 \mathrm{~nm}$ is the interadsorbate distance) because our microscopic approach is applicable only when the wave length of the electromagnetic field is larger than double average distance between the ad- sorbates. In comparison, the friction between two clean surface at separation $d=1 \mathrm{~nm}$ is seven order of magnitude smaller. At $d=1 \mathrm{~nm}$ the friction coefficient $\Gamma$ for an atomic force microscope tip with $R \sim 1 \mu \mathrm{m}$ is $\sim 10^{-12} \mathrm{kgs}^{-1}(\gamma$ $\sim 10^{3} \mathrm{kgs}^{-1} \mathrm{~m}^{-2}$; see Fig. 5); this is of the same order of magnitude as the observed friction. ${ }^{7}$

\section{SUMMARY AND CONCLUSION}

We have calculated the van der Waals friction between two flat surfaces for normal relative motion and have found a drastic difference in the comparison with parallel relative motion. This difference is connected with a resonance condition, produced by the multiple scattering of the electromagnetic waves from the opposite surfaces. In the case of a sharp resonance, the normal relative motion gives a much larger contribution to the friction, as compared to the parallel relative motion.

We have studied in detail the friction between two good conductors and have found that for normal relative motion, even for very small distances the main contribution to friction comes from the retardation effects. We have shown that nonlocal optic effects are very important for the $p$-wave contribution to the friction for parallel relative motion and much less important for normal relative motion. For the $s$-wave contribution, the nonlocal optic effects are unimportant for both directions of relative motion.

In the case of van der Waals friction we have found that for distances between the bodies $\sim 100 \AA$, for good conductors with a high free electrons concentration, the main contribution to the friction is associated with the $s$-polarized electromagnetic waves. For $d<100 \AA$ this mechanism gives a friction coefficient per unit area $\gamma \sim 10^{-5} \mathrm{kgs}^{-1} \mathrm{~m}^{-2}$, nearly independent of the distance $d$, while for $d>100 \AA$ the friction coefficient $\gamma$ depends on distance as $d^{-2}$. For an atomic force microscope tip with the effective surface area $A_{\text {eff }} \approx 10^{-14} \mathrm{~m}^{2}$, we got the friction coefficient $\Gamma \sim \gamma A_{\text {eff }}$ $\sim 10^{-19} \mathrm{kgs}^{-1}$ for $d<100 \AA$. When the concentration of electrons decreases, the $s$ contribution to the friction decreases while the $p$ contribution increases. At $d=100 \AA$ and with the electron lifetime $\tau=4 \times 10^{-14} \mathrm{~s}$, the $p$ contribution reaches a maximum $\gamma_{\max } \sim 10^{-4} \mathrm{kgs}^{-1} \mathrm{~m}^{-2}$ at the electron concentration $n \sim 10^{22} \mathrm{~m}^{-3}$, which corresponds to the conductivity $\sigma \sim 1(\Omega \mathrm{m})^{-1}$.

We have shown that the van der Waals friction can be enhanced by several orders of magnitude in the case of resonant photon tunneling between low-frequency surface plasmon modes and adsorbate vibrational modes. In the case of friction for two $\mathrm{Cu}(100)$ surfaces covered by a low concentration of potassium atoms at $d=10 \AA$ we have found the friction of the same order of magnitude as it was observed in experiment. ${ }^{7}$ However, the distance dependence in this case is stronger than observed in Ref. 7. Further experiments with well defined tips and samples must be performed to elucidate different energy dissipation mechanisms in the noncontact friction. The results obtained in this paper should have a broad application in noncontact friction microscopy, and in the design of new tools for studying adsorbate vibrational dynamics and optical properties of surface plasmons. 


\section{ACKNOWLEDGMENTS}

A.I.V acknowledges financial support from DFG and the Russian Foundation for Basic Research (Project No. 01-02-
16202). B.N.J.P. acknowledges financial support from BMBF. We thank R.O. Jones for help in the numerical calculations.

\section{APPENDIX}

After substituting Eqs. (3) and (5) into formula (20), we obtain, to linear order in vibrational coordinate $u_{0}$ and frequency $\omega_{0}$,

$$
\begin{aligned}
\sigma_{z z}= & \frac{1}{4 \pi} \int_{0}^{\infty} d \omega \int \frac{d^{2} q}{(2 \pi)^{2}}\left[\frac{p}{q^{2}}\left[\left(p+p^{*}\right)\left(\left\langle\left|w_{0 z}\right|^{2}\right\rangle+\left\langle\left|v_{0 z}\right|^{2}\right\rangle\right)+\left(p-p^{*}\right)\left(\left\langle w_{0 z} v_{0 z}^{*}\right\rangle+\text { c.c. }\right)\right]\right. \\
& +\left(\frac{c}{\omega}\right)^{2} p\left[\left(p+p^{*}\right)\left(\left\langle\left|w_{0 y}\right|^{2}\right\rangle+\left\langle\left|v_{0 y}\right|^{2}\right\rangle\right)+\left(p-p^{*}\right)\left(\left\langle w_{0 y} v_{0 y}^{*}\right\rangle+\text { c.c. }\right)\right] \\
& +\left(\frac{p^{+}}{q^{2}}\left[\left(p+p^{*}\right)\left(\left\langle w_{1 z} w_{0 z}^{*}\right\rangle+\left\langle v_{1 z} v_{0 z}^{*}\right\rangle+\text { c.c. }\right)\left(p-p^{*}\right)\left(\left\langle w_{1 z} v_{0 z}^{*}\right\rangle+\left\langle v_{1 z} w_{0 z}^{*}\right\rangle+\text { c.c. }\right)\right]\right. \\
& \left.\left.+\frac{c^{2}}{\omega\left(\omega+\omega_{0}\right)} p^{+}\left[\left(p+p^{*}\right)\left(\left\langle w_{1 y} w_{0 y}^{*}\right\rangle+\left\langle v_{1 z} v_{0 z}^{*}\right\rangle+\text { c.c. }\right)+\left(p-p^{*}\right)\left(\left\langle w_{1 y} v_{0 y}^{*}\right\rangle+\left\langle v_{1 y} w_{0 y}^{*}\right\rangle+\text { c.c. }\right)\right]\right) e^{-i \omega_{0} t}\right] .
\end{aligned}
$$

From Eqs. (21) and (A1) it follows that the friction coefficient is determined by the formula

$$
\begin{aligned}
\gamma_{\perp}= & \frac{1}{4 \pi u_{0} i} \int_{0}^{\infty} d \omega_{0} \int \frac{d^{2} q}{(2 \pi)^{2}}\left[\frac{\partial}{\partial \omega_{0}}\left(\frac{p^{+}}{q^{2}}\left[\left(p+p^{*}\right)\left(\left\langle w_{1 z} w_{0 z}^{*}\right\rangle+\left\langle v_{1 z} v_{0 z}^{*}\right\rangle-\text { c.c. }\right)\left(p-p^{*}\right)\left\langle w_{1 z} v_{0 z}^{*}\right\rangle+\left\langle v_{1 z} w_{0 z}^{*}\right\rangle-\text { c.c. }\right)\right]\right. \\
& \left.\left.+\frac{c^{2}}{\omega\left(\omega+\omega_{0}\right)} p^{+}\left[\left(p+p^{*}\right)\left(\left\langle w_{1 y} w_{0 y}^{*}\right\rangle+\left\langle v_{1 z} v_{0 z}^{*}\right\rangle-\text { c.c. }\right)+\left(p-p^{*}\right)\left(\left\langle w_{1 y} v_{0 y}^{*}\right\rangle+\left\langle v_{1 y} w_{0 y}^{*}\right\rangle-\text { c.c. }\right)\right]\right)\right]_{\omega_{0}=0} .
\end{aligned}
$$

Using Eqs. (12)-(16), (18) and (19), we get

$$
\begin{aligned}
& \frac{1}{q^{2}} \frac{\partial}{\partial \omega_{0}}\left[p^{+}\left(p+p^{*}\right)\left(\left\langle w_{1 z} w_{0 z}^{*}\right\rangle+\left\langle v_{1 z} v_{0 z}^{*}\right\rangle\right)-\text { c.c. }\right]_{\omega_{0}=0} \\
& =2 i u_{0}\left(n(\omega)+\frac{1}{2}\right) \frac{\partial}{\partial \omega}\left[p^{2} \frac{\left(1\left|-R_{1 p} R_{2 p}\right|^{2}\right)^{2}+\left|\left(1-\left|R_{1 p}\right|^{2}\right) R_{2 p} e^{i p d}+\left(1-\left|R_{2 p}\right|^{2}\right) R_{1 p}^{*} e^{-i p d}\right|^{2}}{\left|\Delta_{p}\right|^{4}}\right], \\
& \frac{1}{q^{2}} \frac{\partial}{\partial \omega_{0}}\left[p^{+}\left(p-p^{*}\right)\left(\left\langle w_{1 z} v_{0 z}^{*}\right\rangle+\left\langle v_{1 z} w_{0 z}^{*}\right\rangle-\text { c.c. }\right]_{\omega_{0}=0}\right. \\
& =8 i u_{0}\left(n(\omega)+\frac{1}{2}\right) \frac{\partial}{\partial \omega}\left(\frac { p ^ { 2 } } { | \Delta _ { p } | ^ { 4 } } \left[\left(\operatorname{Im} R_{1 p}+e^{-2|p| d}\left|R_{1 p}\right|^{2} \operatorname{Im} R_{2 p}\right)\left(\operatorname{Im} R_{2 p}+e^{-2|p| d}\left|R_{1 p}\right|^{2} \operatorname{Im} R_{2 p}\right)\right.\right. \\
& \left.\left.+e^{-2|p| d} \operatorname{Im}\left(R_{1 p} R_{2 p}\right)^{2}\right] e^{-2|p| d}\right) \text {. }
\end{aligned}
$$

Other similar expressions for the $s$-wave contribution can be obtained from Eqs. (A3) and (A4) by replacement of the reflection amplitude $R_{p}$ for the $p$-polarized wave by the reflection amplitude $R_{s}$ for the $s$-polarized wave. After substituting Eqs. (A3) and (A4), and similar expressions for $s$-polarized waves, in Eq. (A2) we get formulas (22) and (23) for the friction coefficient for normal relative motion.

${ }^{1}$ T.J. Gramila, J.P. Eisenstein, A.H. MacDonald, L.N. Pfeiffer, and K.W. West, Phys. Rev. Lett. 66, 1216 (1991); Surf. Sci. 263, 446 (1992).

${ }^{2}$ T.J. Gramila, J.P. Eisenstein, A.H. MacDonald, L.N. Pfeiffer, and K.W. West, Phys. Rev. B 47, 12957 (1993); Physica B 197, 442 (1994).

${ }^{3}$ U. Sivan, P.M. Solomon, and H. Shtrikman, Phys. Rev. Lett. 68,
1196 (1992).

${ }^{4}$ A.I. Volokitin and B.N.J. Persson, J. Phys.: Condens. Matter 13, 859 (2001).

${ }^{5}$ I. Dorofeyev, H. Fuchs, G. Wenning, and B. Gotsmann, Phys. Rev. Lett. 83, 2402 (1999).

${ }^{6}$ B. Gotsmann and H. Fuchs, Phys. Rev. Lett. 86, 2597 (2001).

${ }^{7}$ B.C. Stipe, H.J. Mamin, T.D. Stowe, T.W. Kenny, and D. Rugar, 
Phys. Rev. Lett. 87, 096801 (2001).

${ }^{8}$ H.J. Mamin and D. Rugar, Appl. Phys. Lett. 79, 3358 (2001).

${ }^{9}$ P.M. Hoffmann, S. Jeffery, J.B. Pethica, H.Özgür Özer, and A. Oral, Phys. Rev. Lett. 87, 265502 (2001).

${ }^{10}$ I.E. Dzyaloshinskii, E.M. Lifshitz, and L.P. Pitaevskii, Adv. Phys. 10, 165 (1961).

${ }^{11}$ J.A. Sidles et al., Rev. Mod. Phys. 67, 249 (1995).

${ }^{12}$ G.P. Berman et al., Phys. Rev. B 61, 14694 (2000).

${ }^{13}$ N. Arkani-Hamed, S. Dimopoulos, and G. Dvali, Phys. Lett. B 429, 263 (1998); Sci. Am. 283, 62 (2000).

${ }^{14}$ U. Mohideen and A. Roy, Phys. Rev. Lett. 81, 4549 (1998).

${ }^{15}$ A.I. Volokitin and B.N.J. Persson, J. Phys.: Condens. Matter 11, 345 (1999); Phys. Low-Dimens. Semicond. Struct. 7/8, 17 (1998).

${ }^{16}$ B.N.J. Persson and A.I. Volokitin, Phys. Rev. Lett. 84, 3504 (2000)

${ }^{17}$ A.I. Volokitin and B.N.J. Persson, Phys. Rev. B 63, 205404
(2001); Phys. Low-Dimens. Semicond. Struct. 5/6, 151 (2001).

${ }^{18}$ A.I. Volokitin and B.N.J. Persson, Phys. Rev. B 65, 115419 (2002).

${ }^{19}$ A. Krishnan et al., Opt. Commun. 200, 1 (2001).

${ }^{20}$ K.L. Kliever and R. Fuchs, Phys. Rev. 172, 607 (1968); R. Fuchs and K.L. Kliever, ibid. 186, 905 (1969).

${ }^{21}$ N.D. Mermin, Phys. Rev. B 1, 2362 (1970).

${ }^{22}$ E.M. Lifshitz and L.P. Pitaevskii, Physical Kinetics (Pergamon, Oxford, 1981).

${ }^{23}$ U. Hartmann, Phys. Rev. B 42, 1541 (1990); 43, 2404 (1991).

${ }^{24}$ P. Johansson and P. Apell, Phys. Rev. B 56, 4159 (1997).

${ }^{25}$ E.D. Palik, Handbook of Optical Constants of Solids (Academic, San Diego, CA, 1985).

${ }^{26}$ D.C. Langreth, Phys. Rev. B 39, 10020 (1989).

${ }^{27}$ B.N.J. Persson and A.I. Volokitin, Surf. Sci. 310, 314 (1994).

${ }^{28}$ P. Senet et al., Chem. Phys. Lett. 299, 389 (1999). 